\title{
RISK FACTORS AND GINGIVAL INFLAMMATION IN CHILDREN AGED 10 TO 14 YEARS - AN EPI- DEMIOLOGICAL STUDY
}

\author{
Hristina Tankova ${ }^{1}$, Nadezhda Mitova ${ }^{1}$, Maya Rashkova ${ }^{1}$, Hristina Popova ${ }^{2}$ \\ 1) Department of Pediatric Dentistry, Faculty of Dental Medicine, Medical Uni- \\ versity - Sofia, Bulgaria. \\ 2) Department of Periodontology, Faculty of Dental Medicine, Medical Uni- \\ versity - Sofia, Bulgaria.
}

\section{SUMMARY}

Aim: The aim of the present study is an epidemiological study of risk factors and the prevalence of periodontal disease in children aged 10-14 years.

Materials and methods: The subject of the study were 457 children. All were clinically examined, and the data were recorded on a specially designed card. Each child was assessed for risk periodontal profile in 4 levels, which includes: assessment of oral hygiene habits; registration of dental status, reporting of carious lesions and obturations on surfaces dangerous for periodontal health (proximal, cervical); the presence of orthodontic anomalies and other risk factors; assessment of the plaque index of Silness-Loe (PLSL); assessment of gingival index according to Loe and Silness (GILS)

Results: Carious lesions at risk for gingival inflammation surfaces are registered in $59 \%$ of children, at risk for periodontal health fillings in $32 \%$ and orthodontic anomalies in $37 \%$. The studied children showed relatively good oral hygiene (PLSL- $0.43 \pm 0.28$ ), which improved with age, with the largest amount of plaque observed on the proximal and vestibular surfaces. Over $88 \%$ of children have mild gingival inflammation (GILS-0.56 \pm 0.42 ), mainly affecting the front teeth in both jaws.

Conclusions: Gingival inflammation in 10-14 year old children covers more than half of the studied children, with mild forms of inflammation predominating with a relatively low oral hygiene index. 2/3 of registered caries and $1 / 3$ of the fillings have a localization which presents a risk for periodontal health. Orthodontic anomalies are the next most serious risk factor for plaque accumulation and gingival inflammation.

Keywords: gingival inflammation, risk factors, periodontal indices,

\section{INTRODUCTION}

The period of mixed dentition is characterized by an increased risk of periodontal disease. This is a period of unstable oral hygiene habits, risky nutrition for plaque accumulation, orthodontic anomalies, hormonal changes related to the sexual growth of children and the formation of subgingival microflora immediately after the tooth eruption of permanent children's teeth.

The modern classification of periodontal diseases focuses on local risk factors and defines periodontal pathology primarily as site specific condition. This new concept is related to the specification of the local factor of the inflammatory reaction in the gingiva in the context of the overall gingival status of the patient $[1,2]$.

As children grow and develop, the composition of the subgingival biofilm changes. It is influenced by the immune system, hormonal status, tooth eruption.

Various epidemiological studies have shown that gingivitis is prevalent in children and adolescents worldwide, and the results obtained by various authors show that about half of children in early adolescence have plaqueassociated gingivitis $[3,4,5,6]$. Other author groups found a lower relative share of these diseases, which is probably due to the use of different indices to objectify the gingival status in the study of children [7]. For epidemiological purposes, according to the latest consensus among leading periodontists, gingival inflammation is defined as a condition in which BOP (bleeding on probing) involving more than $10 \%$ of the gingiva and a probing depth less than or equal to $3 \mathrm{~mm}$ is observed [2].

\section{AIM}

The aim of the present study is an epidemiological study of risk factors and the prevalence of periodontal disease in children aged 10-14 years.

\section{MATERIAL AND METHODS}

The subject of the study were 457 children aged 10 to 14 years.

The study was conducted in two Sofia schools. The children were evenly divided into five groups according to age - $10,11,12,13$, and 14 years old.

The criteria for inclusion of children in the study were:

- informed consent signed by the parents for participation in the scientific research approved by the Research Ethics Committee with protocol No.12 / 14.05.2020;

- lack of systemic diseases or drug therapy; 
- lack of non-removable orthodontic appliances;

\section{Methodology}

All the children were clinically examined, and the data were recorded on a specially designed card. The clinical trial was performed by an examiner in a clinical office. Disposable examination instruments and a mechanical periodontal probe were used, and the data were recorded with the participation of a dental assistant.

Each child was assessed for the risk of periodontal pathology at 4 levels:

- Level 1 - Anamnestic data for systemic pathology, risk for periodontal health; behavioral factors - assessment of oral hygiene procedures (frequency of brushing);

- Level 2 - oral environment - assessment of plaque accumulation by plaque index according to Silness and Loe;

- Level - teeth and gingiva - registration of dental status by including carious lesions with diagnostic threshold D3 and reporting of carious lesions and fillings risky for periodontal health (proximal, cervical); the presence of orthodontic anomalies; assessment of the width of the attached gingiva in the lower front and the presence of gingival recessions as a risk factor for periodontal destruction;

- Level 4 - microenvironment - assessment of gingival inflammation by gingival index according to Loe and Silness;

The registering of the orthodontic anomalies was performed according to the criteria for orthodontic deviations accepted in the Department of Orthodontics of FDM Sofia.

To estimate the width of the attached gingiva was adopted the norm of $2 \mathrm{~mm}$ in the area of the lower front teeth and any deviation below $2 \mathrm{~mm}$ was accepted as a reduced width.

Gingival recessions were registered in all cases in which withdrawal of the marginal gingival tissues apically from the enamel-cement border of the examined teeth was observed.

The reading of the Silness and Loe plaque index and the Loe and Silness gingival index [8] was performed using a mechanical periodontal probe (WHO 621) on all examined permanent teeth at four points - medial, vestibular, distal and oral, the classical methodology of the index is observed.

The calculation of the index for the patient is the sum of all reported values divided by the number of examined points. The same calculation was performed in sextants.

SPSS Windows, version 19.0, was used for statistical data processing.

\section{RESULTS}

\section{Level 1. Oral hygiene habits}

The following figure shows the frequency of oral hygiene procedures in the examined children (Figure 1).

Fig. 1. The frequency of oral hygiene procedures in the examined children

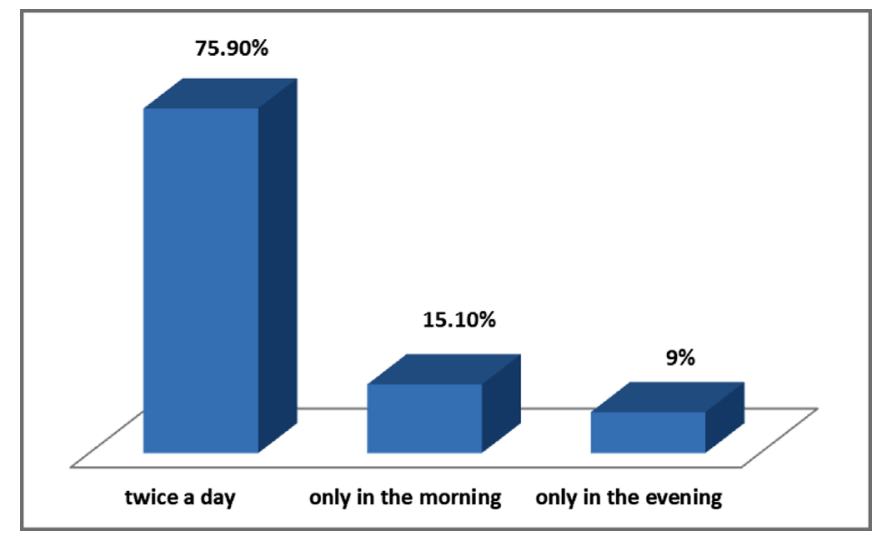

The figure shows that in terms of brushing frequency, over $2 / 3$ of children brush their teeth twice a day, while the remaining $1 / 3$ apply their oral hygiene procedures once a day. This gives reason to believe that the vast majority of children between the ages of 10 and 14 have properly developed oral hygiene habits.

\section{Level 2. Oral hygiene status}

The following table shows the mean Silness-Loe Plaque Index (PLSL) in the different age groups (Table 1).

Table 1. Mean value of Plaque Index by Silness-Loe (PLSL) in different age groups

\begin{tabular}{|l|c|l|}
\hline \multirow{2}{*}{ Age } & \multicolumn{2}{|c|}{ Plaque indexPLSL } \\
\cline { 2 - 3 } 10 years old & $\mathrm{N}$ & Mean \pm SD \\
\hline 11 years old & 99 & $0.61 \pm 0.34$ \\
\hline 12 years old & 97 & $0.47 \pm 0.22$ \\
\hline 13 years old & 92 & $0.42 \pm 0.24$ \\
\hline 14 years old & 80 & $0.30 \pm 0.21$ \\
\hline Total & 457 & $0.43 \pm 0.28$ \\
\hline ANOVA & $\mathrm{T}=23.395 \mathrm{p}<0.05$ \\
\hline
\end{tabular}

The table shows that the mean value of the SilnessLoe plaque index in all examined children is $0.43 \pm 0.28$, which shows that the children have good oral hygiene. The age distribution shows the highest value in 10-year-old children and a progressive decrease in value with increasing age.

The following table shows the distribution of the Silness-Loe plaque index by surfaces (Table 2). 
Table 2. Distribution of plaque index Silness-Loe (PLSL) on surfaces

\begin{tabular}{|l|l|l|}
\hline Localization ofPLSL & Mean \pm SD & One sample T test \\
\hline Vestibular & $0.55 \pm 0.51$ & $\mathrm{~T}=55.455 \mathrm{p}<0.05$ \\
\hline Proximal & $0.56 \pm 0.51$ & $\mathrm{~T}=57.802 \mathrm{p}<0.05$ \\
\hline Oral & $0.09 \pm 0.25$ & $\mathrm{~T}=19.682 \mathrm{p}<0.05$ \\
\hline
\end{tabular}

The values of the plaque index on the proximal and vestibular surfaces are respectively $0.56 \pm 0.51$ and $0.55 \pm$ 0.51 , while on the oral surfaces, the value decreases repeatedly and is $-0.09 \pm 0.25$ ( $\mathrm{p}<0.05)$, which is the probable reason for the relatively low average values of the plaque index.
Level 3. - risk factors for periodontal health (teeth, occlusion, gingiva)

3.1 Carious lesions and fillings risk for periodontal health

The average caries presence, represented by the DMF $(\mathrm{T}+\mathrm{t})$ - index, of the examined children is 2.33 teeth per child. With age, there is a progressive decrease in this value, which is explained by the gradual transition from mixed to permanent dentition.

The following table shows the relative share of carious lesions and fillings, which present a risk for periodontal health (Table 3).

Table 3. Carious lesions and fillings risky for periodontal health

\begin{tabular}{|l|c|c|c|c|c|c|}
\hline \multirow{2}{*}{$\begin{array}{l}\text { Affected } \\
\text { by caries }\end{array}$} & \multicolumn{2}{|c|}{ Non risky } & \multicolumn{2}{c|}{ Risky } & \multicolumn{2}{c|}{ Total } \\
\cline { 2 - 7 } & $\mathrm{n}$ & $\%$ & $\mathrm{n}$ & $\%$ & $\mathrm{n}$ & $\%$ \\
\hline Carious lesions & 186 & 41 & 263 & 59 & 449 & 100 \\
\hline Fillings & 415 & 68 & 195 & 32 & 610 & 100 \\
\hline
\end{tabular}

The table shows that the total number of carious lesions in all examined children is 449 , of which more than half $-59 \%$ are at risk sites related to their periodontal health. There are a total of 610 obturated teeth, of which only $32 \%$ are at risk sites for periodontal health.

\subsection{Orthodontic anomalies}

The following figure shows the relative proportion of orthodontic anomalies by age (Figure 2).

Fig. 2. Relative share of orthodontic anomalies by age

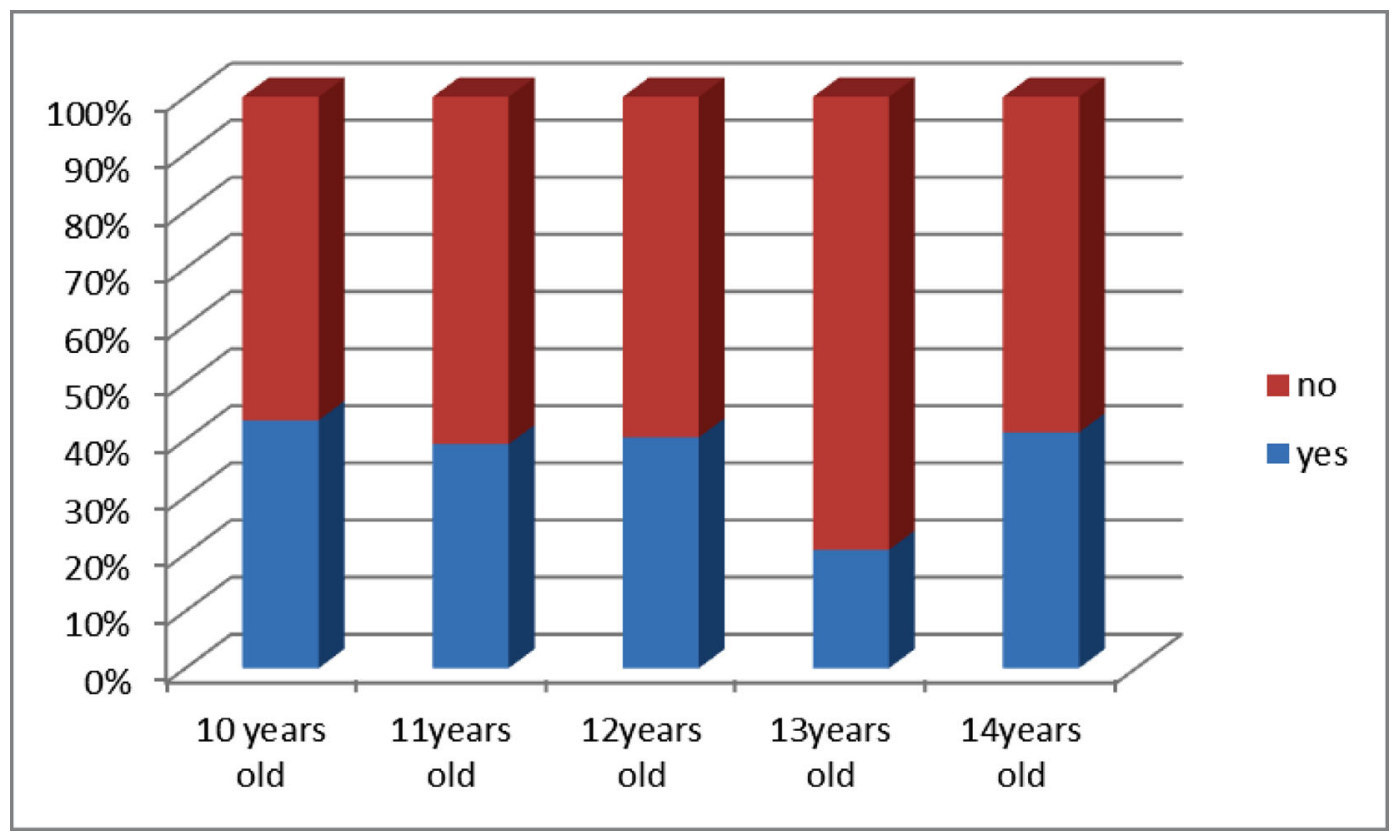

Figure 2 shows that in a little over $1 / 3$ of the examined children, the presence of an orthodontic anomaly is established, as the percentage of 10-year-olds is the high- est among the children with malocclusions.

The following figure shows the distribution of different types of dental jaw deformities (Figure 3). 
Fig. 3. Distribution of different types of orthodontic anomalies

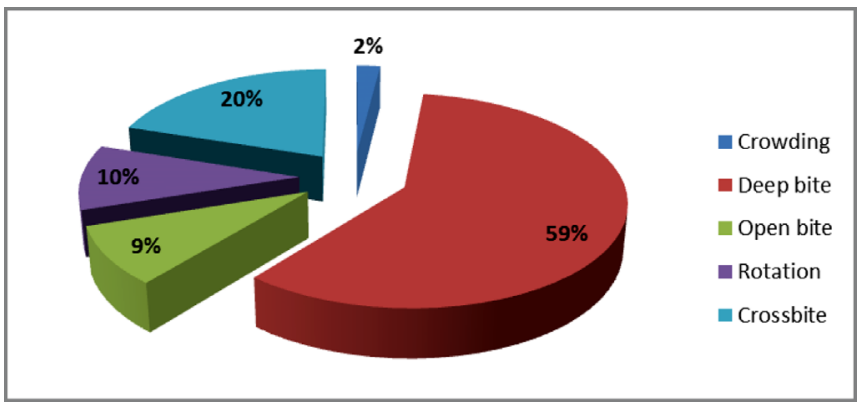

It is clear from Figure 3 that more than half of all examined children $(58.6 \%)$ have a deep bite. The remaining orthodontic anomalies occur in $1 / 3$ of the examined children.

\subsection{Gingival recessions}

The following figure shows the relative share of gingival recessions in all examined children (Figure 4).

Fig. 4. Relative share of gingival recessions in the examined children

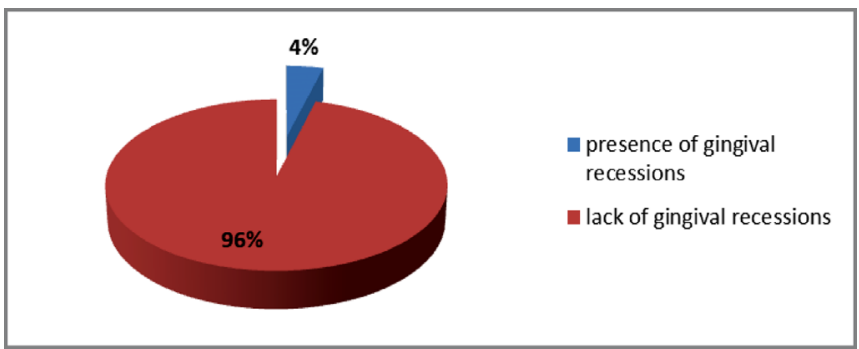

The figure shows that only $4 \%$ of all examined children are diagnosed with gingival recession. Over $95 \%$ of children do not have a clinically established recession.

\subsection{Width of the attached gingiva}

The following figure shows the relative proportion of reduced width of the attached gingiva in the area of the lower front in all examined children (Figure 5).

Fig. 5. Relative share of width of the attached gingiva in the area of the lower front teeth

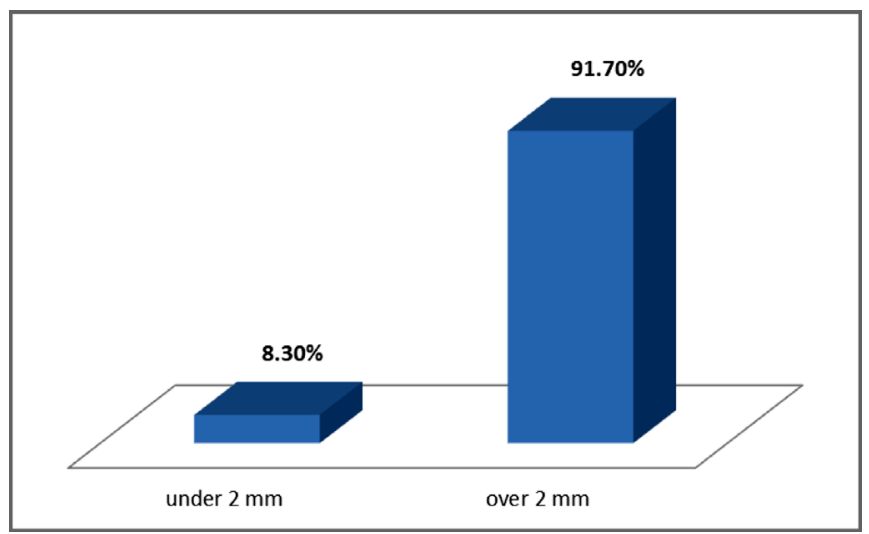

It is clear from the figure that only $1 / 10$ of all examined children have a clinically established reduced width of the attached gingiva.

Level 4. Gingival status of the examined children

The following table presents the mean values of gingival inflammation according to Loe-Silness, in the different age groups (Table 4).

Table 4. Mean gingival index Loe-Silness (GI LS) by age

\begin{tabular}{|l|c|c|}
\hline \multirow{2}{*}{ Age } & \multicolumn{2}{|c|}{ Gingival indexGI LS } \\
\cline { 2 - 3 } 10 years old & $\mathrm{n}$ & Mean \pm SD \\
\hline 11 years old & 99 & $0.58 \pm 0.56$ \\
\hline 12 years old & 89 & $0.63 \pm 0.28$ \\
\hline 13 years old & 92 & $0.66 \pm 0.39$ \\
\hline 14 years old & 80 & $0.30 \pm 0.30$ \\
\hline Total & 457 & $0.56 \pm 0.42$ \\
\hline ANOVA & $\mathrm{T}=10.666 \mathrm{p}<0.05$ \\
\hline
\end{tabular}

The highest value is registered in 12-year-old children - $0.66 \pm 0.39$, and the lowest - in 14-year-olds, where the value drops twice.

The following figure shows the distribution of children according to the severity of the gingival Loe-Silness index (Figure 6).

Fig. 6 Distribution of children according to the degree of severity of the gingival index Loe-Silness

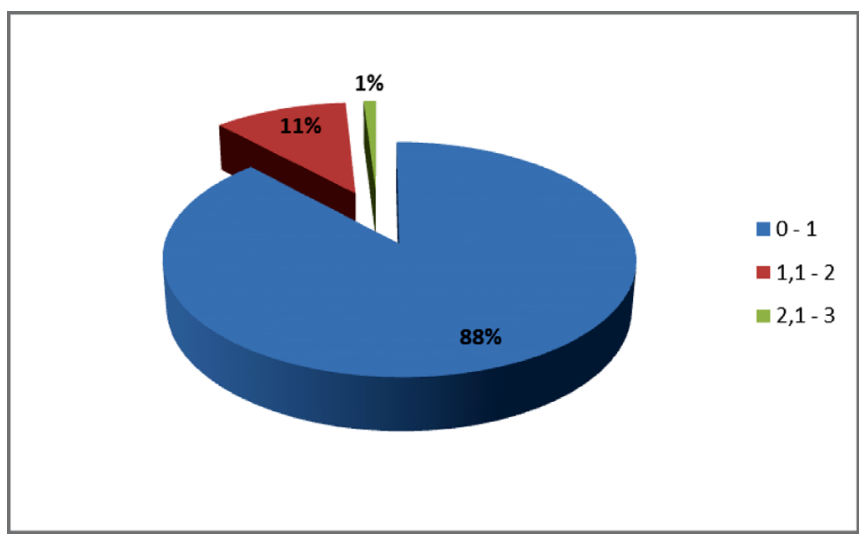

The figure shows that $88 \%$ of all examined children have a value of gingival index between 0 and 1 . Only $11 \%$ have a value of the index between 1 and 2, which indicates moderate inflammation, and less than $1 \%$ have values above 2 .

4.1. Correlation between Silness-Loe plaque index and Loe-Silness gingival index, by sextants

The following table presents the comparison between plaque and gingival index by sextants (Table 5). 
Table 5. Comparison between plaque index SilnessLoe and gingival index Loe-Silness by sextants

\begin{tabular}{|l|l|l|}
\hline \multirow{2}{*}{ Sextants } & PL SL & GI LS \\
\cline { 2 - 3 } & Mean \pm SD & Mean \pm SD \\
\hline I sextant & $0.36 \pm 0.39$ & $0.18 \pm 0.44$ \\
\hline II sextant & $0.59 \pm 0.40$ & $0.98 \pm 0.74$ \\
\hline III sextant & $0.72 \pm 3.69$ & $0.20 \pm 0.50$ \\
\hline IV sextant & $0.28 \pm 0.37$ & $0.11 \pm 0.37$ \\
\hline V sextant & $0.50 \pm 0.36$ & $1.04 \pm 0,77$ \\
\hline VI sextant & $0.28 \pm 0.34$ & $0.13 \pm 0.39$ \\
\hline ANOVA & $\mathrm{T}=6.060 \mathrm{p}=0.000$ & $\mathrm{~T}=282.768 \mathrm{p}=0.000$ \\
\hline
\end{tabular}

The table shows that a larger amount of plaque and, respectively, more severe gingival inflammation is observed in the front teeth of the lower and upper jaw (II and Vsextant). It was found that in the area of molars and premolars in the upper jaw (I and III sextant), more plaque is retained compared to the same teeth in the lower jaw, which leads to more pronounced inflammation in these sextants.

The following figure shows pearson correlation between plaque index Silness-Loe and gingival index LoeSilness by sextants (Figure 7).

Fig. 7 Pearson correlation between plaque and gingival index (Silness-Loe / Loe-Silness) by sextants

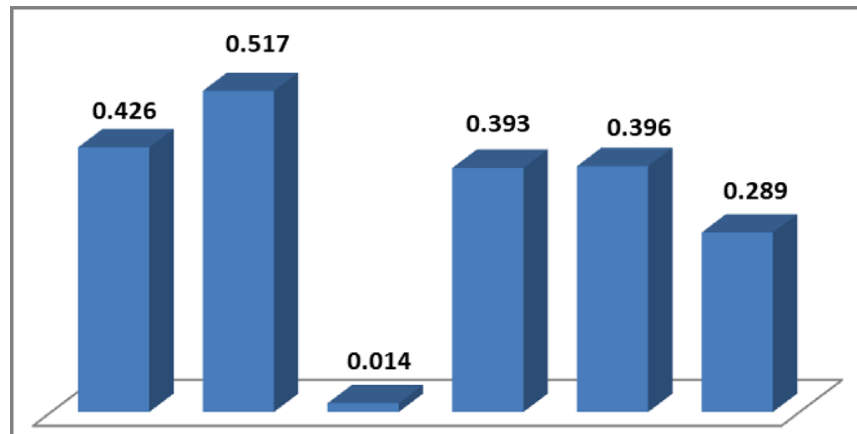

I sextant II sextant III sextant IV sextant V sextant VI sextant correlation coefficient

From the figure it is visible that there is a medium correlation between plaque accumulation and gingival inflammation in all sextants except III. The high plaque accumulation in the third sextant does not correspond to the gingival inflammation in the same area, which may be due to food intake immediately before the examination.

\section{DISCUSSION}

According to our study, it is clear that the oral hygiene habits of the studied children are relatively good, with an average value of the plaque index of Silness and Loe is $0.43 \pm 0.28$, which is an indicator of good oral hygiene. It is well known that subjects performing high oral hygiene standards at daily intervals will maintain gingival health longer in lifespan [9]. Twice the lower values of plaque accumulation on the oral surfaces compared to the vestibular and proximal dental surfaces is the probable reason for the relatively low average values of the registered plaque index, which are below one.

From the present study, it is clear that the transition from mixed to permanent dentition is characterized by gradual improvement of oral hygiene procedures, reduction of the number of carious lesions on risk surfaces due to their physiological change, which inevitably leads to proportional reduction of plaque index Silness-Loe with age.

In the study, it was found that the relative proportion of carious lesions leading to risk for periodontal health (proximal, cervical) is 59\%, and the number of these carious lesions decreases with age. The presence of such lesions is associated with the tendency to retain more plaque near the gingival margin, which is a major etiological factor in periodontal pathology. The effect of irregularly contoured fillings located on surfaces that are dangerous for periodontal health is similar [10]. We found that in the examined children, the relative share of these fillings is $32 \%$.

Orthodontic anomalies are the third most common oral pathology after caries and periodontal diseases and are considered a risk factor for the accumulation of dental plaque and its difficulty in removal [11]. Our study found that $1 / 3$ of the examined children have orthodontic anomalies, most of them are with a deep bite. A group of scientists have found that the chewing forces that are generated in the presence of a deep bite can accelerate the development of periodontal disease. This type of malocclusion usually results in the retention of large amounts of food that remains on the vestibular surface of the lower frontal teeth, which in combination with unestablished oral hygiene habits in childhood could be considered a risk factor in periodontal pathology [12].

The present study established that the percentage of children with gingival recessions was insignificant (4\%), and it can be concluded that the impact of gingival recessions in periodontal pathology in children aged 10 to 14 years is minimal and is rather an exception. On the other hand, the presence of a reduced width of the attached gingiva is a risk indicator for both future gingival recessions and localized periodontal destruction. Our study found a prevalence of $8 \%$ reduced width of the attached gingiva in all children studied. Despite the small number of children with reduced width of the attached gingiva, we believe that this indicator is extremely important for the prevention of future periodontal complications and should be considered in orthodontic treatment of lower frontal teeth to prevent the process from becoming more severe.

The present study found that according to the Loe and Silness gingival index used, nearly $90 \%$ of the children studied had mild plaque-associated gingivitis. The values of the gingival index of Loe and Silness are comparable to those of the plaque index of Silness and Loe and show a high correlation between the indices, with higher values of the front teeth in the upper and lower jaw. Similar to our results are obtained by other author teams that use the same indices for evaluation $[13,14,15]$. 


\section{CONCLUSIONS:}

1. The studied children aged 10-14 years show relatively good oral hygiene, which improves with age.

2. The largest amount of plaque is observed on the approximal and vestibular surfaces, which correlates with gingival bleeding.

3. In $59 \%$ of children aged 10-14, carious lesions at risk for gingival inflammation are registered, with $32 \%$ fillings at risk for gingival inflammation and with $37 \%$ of orthodontic anomalies, which may be the cause of increased plaque accumulation.
4. The reduced width of the attached gingiva, as well as the gingival recessions, are insignificant risk factors for periodontal pathology at the age of 10-14 years.

5 . $88 \%$ of children aged 10-14 have mild gingival inflammation, which mainly affects the front teeth in the upper and lower jaw.

The publication is under the Grant 2020 project with CONTRACT No,113 / 24.06.2020. on the topic "Epidemiology, diagnostics, treatment and prevention of plaque-induced gingivitis in childhood and adolescence."

\section{REFERENCES:}

1. Caton JG, Armitage G, Berglundh T, Chapple ILC, Jepsen S, Kornman KS, et al. A new classification scheme for periodontal and peri-implant diseases and conditions - Introduction and key changes from the 1999 classification. $J$ Clin Periodontol. 2018 Jun;45 Suppl 20:S1-S8. [PubMed]

2. Trombelli L, Farina R, Silva CO, Tatakis DN. Plaque-induced gingivitis: Case definition and diagnostic considerations. J Clin Periodontol. 2018 Jun;45(Suppl 20):S44-S67. [PubMed]

3. Albandar JM. Epidemiology and risk factors of periodontal diseases. Dent Clin North Am. 2005 Jul;49(3): 517-32, v-vi. [PubMed]

4. Peycheva S, Apostolova E, Gardjeva P, Peychev Z, Kokova V, Angelov A, et al. Effect of Bulgarian propolis on the oral microflora in adolescents with plaque-induced gingivitis. Rev Bras Farmacogn. 2019 MayJun;29(3):271-277. [Crossref].

5. Rashkova M, Ribagin L, Doganova Ts, Aleksieva V. Children's oral diseases in Rouse region - epidemiological research, a part of the National Program of Prophylaxis of Oral Diseases in Children 0-18 years in Bulgaria. Problems of dental medi- cine. 2011; 37(2):22-31.

6. Nair RA, Jain J, Praveena J, Shashidhar K, Pooja MR, Shetty S. Incidence of Dental Caries and Periodontal Diseases among Adolescents Receiving Orthodontic Treatment in a Dental Institute in Virajpet. $J$ Evol Med Dent Sci. 2021 Apr;10(16):1119.

7. ARCPOH, The University of Adelaide, South Australia. Periodontal diseases in the Australian adult population. Aust Dent J. 2009 Dec;54(4): 390-3. [PubMed]

8. Silness J, Loe H. Periodontal Disease in Pregnancy. II. Correlation Between Oral Hyggiene and Periodontal Condtion. Acta Odontol Scand. 1964 Feb;22:121-35. [PubMed]

9. De David SC, Mario TG, De Freitas GC, Kantorski KZ, Wikesjo UME, Moreira CHC. Correlation between plaque control and gingival health using short and extended oral hygiene intervals. Clin Oral Investig. 2018 Sep;22(7):2593-2597. [PubMed] 10. Murakami S, Mealey BL, Mariotti A, Chapple ILC. Dental plaque-induced gingival conditions. $J$ Periodontol. 2018 Jun;89(Suppl 1):S17-S27. [PubMed]
11. Brito DI, Dias PF, Gleiser R. Prevalence of malocclusion in children aged 9 to 12 years old in the city of nova friburgo, rio de Janeiro State, Brazil. Rev Dent Press Ortodon Ortop Facial. 2009 Nov-Dec;14(6):118-124. [Crossref]

12. Daing A, Jafri Z, Bhardwaj A, Sawai MA, Sultan N, Periodontal lesions associated with deep bite: Report of three cases. Int $\mathrm{J}$ Oral Health Dent. 2018 Jan-Mar;4(1):43-45. [Crossref]

13. Jenkins WM, Papapanou PN. Epidemiology of periodontal disease in children and adolescents. Periodontol 2000. 2001; 26:16-32. [PubMed]

14. Nakagawa S, Fujii H, Machida Y, Okuda K. A longitudinal study from prepuberty to puberty of gingivitis. Correlation between the occurrence of Prevotellaintermedia and sex hormones. J Clin Periodontol. 1994 Nov; 21(10):658-65. [PubMed]

15. McDonald RE, Avery DR. Dentistry for the Child and Adolescent. 7th Edition. Dean JA (Editor). Mosby. September 22, 1999, pp.440-452. [Internet]

Please cite this article as: Tankova H, Mitova N, Rashkova M, Popova H. Risk factors and gingival inflammation in children aged 10 to 14 years - an epidemiological study. J of IMAB. 2021 Oct-Dec;27(4):4092-4097. DOI: https://doi.org/10.5272/jimab.2021274.4092

Received: 27/04/2021; Published online: 12/11/2021

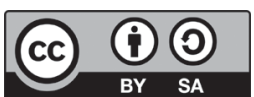

Address for correspondence:

Dr. Hristina Ivanova Tankova-Zlateva

Department of Pediatric Dentistry, Faculty of Dental Medicine, MU - Sofia,

1, GeorgiSofiyski Str., Sofia, Bulgaria.

E-mail: la_svetichi@yahoo.com 